\title{
The Neostriatal Mosaic: III. Biochemical and Developmental Dissociation of Patch-Matrix Mesostriatal Systems
}

\author{
Charles R. Gerfen, ${ }^{1}$ Ken G. Baimbridge, ${ }^{2}$ and Jean Thibault ${ }^{3}$ \\ 'Laboratory of Neurophysiology and Laboratory of Cell Biology, National Institute of Mental Health, Bethesda, Maryland \\ 20892, ${ }^{2}$ Department of Physiology, University of British Columbia, Vancouver, BC, Canada, and ${ }^{3}$ Department of Cellular \\ Biochemistry, Collège de France, Paris
}

In the previous paper (Gerfen et al., 1987) mesostriatal dopaminergic neurons were shown to be subdivided into dorsal and ventral tiers that project to the striatal matrix and patch compartments, respectively. The present study provides experimental evidence that these patch-matrix mesostriatal dopaminergic systems are biochemically and developmentally distinct. A $28 \mathrm{kDa}$ calcium-binding protein (CaBP, or calbindin- $D_{28 \mathrm{kDa}}$ ) is expressed in dorsal tier mesostriatal dopaminergic neurons. The distribution of such neurons, located in the ventral tegmental area, dorsal tier of the substantia nigra pars compacta, and retrorubral area, matches that of dopaminergic neurons that project to the striatal matrix. Dopaminergic neurons that do not express CaBP-those in the ventral tier of the pars compacta and in the pars reticulata-are distributed in a pattern that matches the origin of the dopaminergic projection to the striatal patches. During development, dopaminergic afferents to the striatal patch compartment are in place prior to the development of those to the matrix. Injections of the neurotoxin 6-hydroxydopamine (6-OHDA) into the striatum of newborn rats result in a selective and long-lasting depletion of dopaminergic afferents in the striatal patches. The later-developing matrix projection is relatively spared by such lesions. The distribution of surviving dopaminergic neurons, labeled with tyrosine hydroxylase (TH) immunoreactivity, matches the pattern of dorsal tier neurons previously shown to provide inputs to the matrix. Surviving neurons also express CaBP immunoreactivity and have dendrites that spread mediolaterally, in the plane of the pars compacta. On the other hand, those neurons that project to the patches are selectively lesioned by the neonatal 6-OHDA striatal injections, do not express CaBP, and have dendrites that are directed ventrally into the pars reticulata.

The homogenous appcarance of dopaminergic innervation of the adult striatum is comprised of the interlaced mosaic of patchand matrix-directed systems arising from separate ventral and dorsal sets of midbrain dopaminergic neurons (Gerfen et al., 1987). Several studies suggest that these 2 mesostriatal systems are differentially regulated and/or biochemically or physiolog-

\footnotetext{
Received Dec. 3, 1986; revised Mar. 27, 1987; accepted June 5, 1987.

We wish to thank William G. Benson for excellent technical support. Rachel Saunders assisted in the quantitative analysis.

Correspondence should be addressed to C. R. Gerfen, LCB, NIMH, Bldg. 36, Rm. 3A-17, Bethesda, MD 20892.

0270-6474/87/123935-10\$02.00/0
}

ically distinct. For example, following pharmacologic treatments that deplete dopamine stores in mesostriatal terminals, there is a more rapid replenishment of dopamine in patch than in matrix areas (Olson et al., 1972; Fuxe et al., 1979; Fukui et al., 1986). Also, chronic apomorphine treatment results in an increase in dynorphin immunoreactivity in patch and not matrix striatonigral neurons (Li et al., 1986). The manner in which the patch- and matrix-directed mesostriatal dopaminergic neurons may be differentially regulated by the compartmental organization of striatonigral projections (Gerfen, 1984, 1985) was discussed in the previous paper (Gerfen et al., 1987). In the present study, the 2 mesostriatal dopaminergic systems are shown to be biochemically differentiated by the expression of a $28 \mathrm{kDa}$ calcium-binding protein (calbindin- $\mathrm{D}_{28 \mathrm{kDa}}$, or CaBP; Baimbridge et al., 1982) in the matrix-directed dorsal mesostriatal neurons. This protein has also previously been shown to be expressed in striatal matrix neurons that project to the substantia nigra pars reticulata (Gerfen et al., 1985).

Patch- and matrix-directed mesustriatal systems develop asynchronously, with the dopaminergic inputs to the patches developing prior to those in the matrix (Olson et al., 1972; Tennyson et al., 1972; Butcher and Hodge, 1976; Graybiel et al., 1981; Graybiel, 1984; Moon-Edley and Herkenham, 1984; van der Kooy, 1984). This developmental sequence was examined in the present study by making injections of the neurotoxin 6-hydroxydopamine (6-OHDA) into the striatum of newborn rats at a time when the patch-directed system was in place, and prior to the ingrowth of the bulk of the input to the matrix. Analysis of mesostriatal neurons that survive such lesions, resulting in the relatively selective deafferentation of striatal patches, provides a means of determining the distribution and dendritic morphology of matrix-directed neurons.

\section{Materials and Methods}

Immunohistochemistry. The midbrains of adult Sprague-Dawley rats were processed to localize $28 \mathrm{kDa}$ calcium-binding protein (CaBP; calbindin- $\mathrm{D}_{28} \mathrm{kDa}_{\mathrm{a}}$ ) and tyrosine hydroxylase $(\mathrm{TH})$ by standard immunohistochemical methods. The rats were deeply anesthetized with ether and perfused transcardially with normal physiological saline $(0.9 \% \mathrm{NaCl})$, followed by $500 \mathrm{ml}$ of fixative, containing $4 \%$ formaldehyde (from paraformaldehyde), $20 \mathrm{~mm}$ sodium buffer (pH 7.4), and $100 \mathrm{~mm} \mathrm{NaCl}$. Brains were removed and postfixed for 24-48 $\mathrm{hr}$ and then sectioned with a Vibratome. Sections $30 \mu \mathrm{m}$ thick were collccted in $0.02 \mathrm{M}$ potassium phosphate-buffered saline (KPBS, pH 7.4). Adjacent series of sections through the midbrain were incubated in solutions of KPBS and $2 \%$ normal goat serum (NGS) and $0.5 \%$ Triton X-100, in which were diluted either rabbit antiserum directed against CaBP (1:1000; see Gerfen et al., 1985) or rabbit antiserum directed against TH (1:1500; see Arluison et al., 1984) for $24-48 \mathrm{hr}$ at $4^{\circ} \mathrm{C}$. Sections were then rinsed 3 

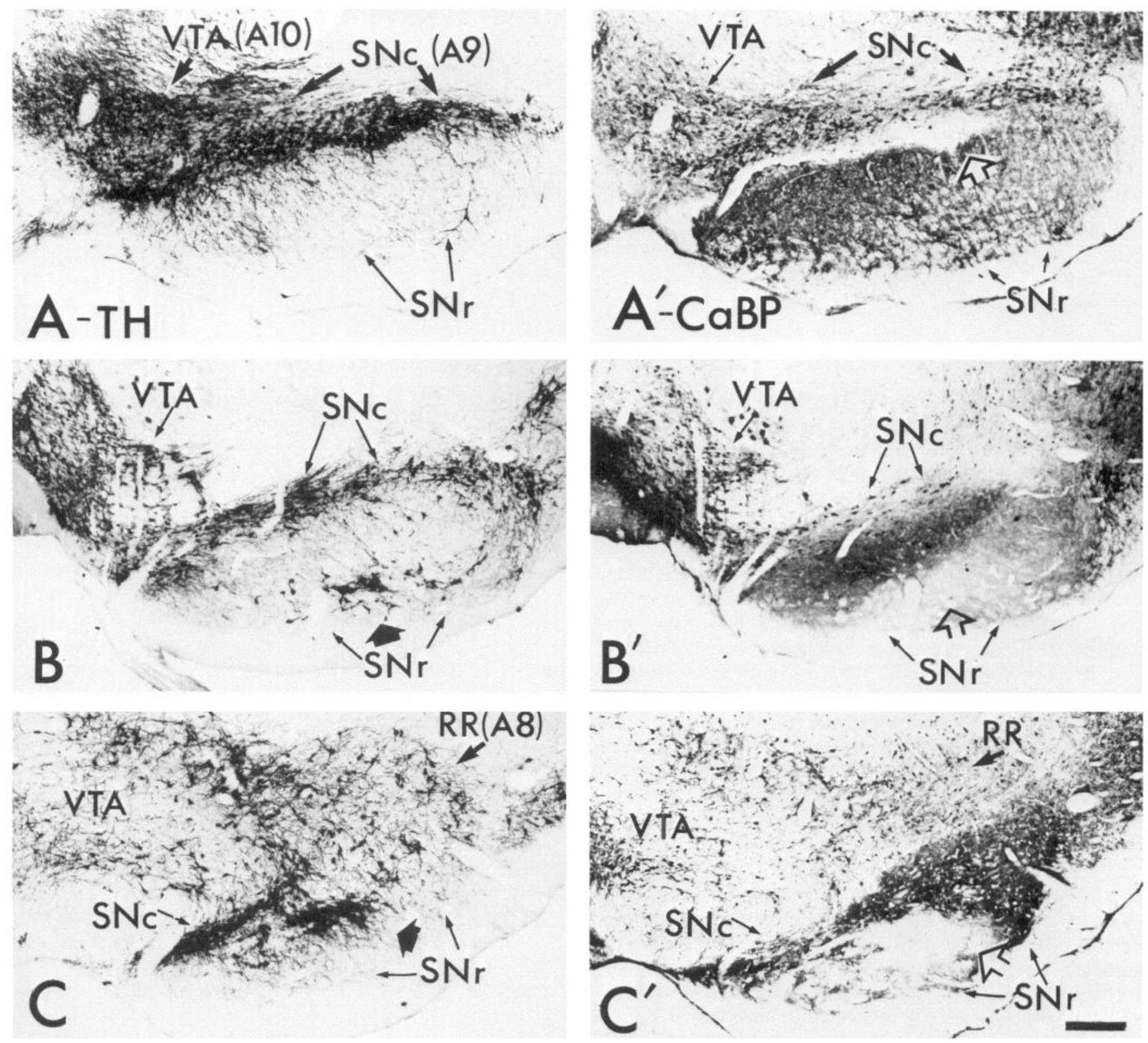

Figure 1. Photographs of adjacent coronal sections through rostral $\left(A, A^{\prime}\right)$, mid $\left(B, B^{\prime}\right)$, and caudal $\left(C, C^{\prime}\right)$ levels of the ventral midbrain show TH-immunoreactive labeling of dopaminergic neurons $(T H ; A-C)$ and calbindin- $\mathrm{D}_{28 \mathrm{kDa}}$-containing neurons and terminals $\left(C a B P ; A^{\prime}-C^{\prime}\right)$. At rostral levels $\left(A, A^{\prime}\right)$, the $\mathrm{A} 10$ dopamine cell group in the ventral tegmental area $(V T A)$ is located medial (to the left) to the A9 dopamine cell group, which at this level is confined to the substantia nigra pars compacta $(S N c)$, dorsal to the pars reticulata $(S N r)$. CaBP immunoreactivity is expressed in many VTA and dorsal tier SNc cells $\left(A^{\prime}\right)$. CaBP-immunoreactive terminals, from striatal matrix neurons, are distributed in the SNr and avoid dopar..ine cell bodies. At mid midbrain levels $\left(B, B^{\prime}\right)$, there is a similar distribution of $A 10$ and A9 dopaminergic and CaBP-immunoreactive neurons, except that there are A9 dopaminergic neurons distributed in the ventral $\mathrm{SNr}$ (broad arrow). At this level, neither ventral tier SNc nor $\mathrm{SNr}$ dopaminergic cells express CaBP immunoreactivity. Also of note is the absence of CaBP-immunoreactive terminals in those parts of the pars reticulata where dopaminergic neurons are located (open arrow, $\left.B^{\prime}\right)$. At caudal levels $\left(C, C^{\prime}\right)$, the VTA is similar to that at other levels in containing a high percentage of $\mathrm{CaBP}$-immunoreactive neurons, and the $\mathrm{A} 8$ dopaminergic cell group, which is evident dorsally in the retrorubral area $(R R)$, similarly has a high percentage of CaBP-immunoreactive neurons. Scale bar, $500 \mu \mathrm{m}$.

times for $5 \mathrm{~min}$ in KPBS and reacted for immunoperoxidase localization of the primary antisera. First, sections were incubated for $60 \mathrm{~min}$ in goat anti-rabbit IgG conjugated to biotin (BRL, Bethesda, MD; diluted $1: 200$ in KPBS plus $\%$ NGS and $0.5 \%$ Trition X-100) and then rinsed 3 times for $5 \mathrm{~min}$. Next, sections were incubated for $60 \mathrm{~min}$ in streptavidin-conjugated HRP (BRL; diluted 1:200 in KPBS) and then rinsed 3 times for $5 \mathrm{~min}$ in KPBS. Sections were then reacted for 5-25 min in a solution containing $0.05 \%$ diaminobenzidine (DAB; Litton Bionetics), $0.004 \%$ hydrogen peroxide diluted in KPBS, which had previously been filtered through a $0.22 \mu \mathrm{m}$ Millipore filter. To stop the re- action, sections were transferred to $10 \%$ formalin for 5-10 min and then rinsed in KPBS before mounting onto chrom-alum-coated slides. After air-drying, the slide-mounted sections were dehydrated and defatted in equal parts of chloroform and methanol twice for $30 \mathrm{~min}$, rehydrated and intensified in $0.005 \%$ osmium tetroxide for $4 \mathrm{hr}$, rinsed for $15 \mathrm{~min}$, dehydrated, and coverslipped out of xylene.

Neonatal 6-OHDA striatal lesions. The forebrains of newborn rat pups (postnatal days $0-8, \mathrm{P} 0-8$ ) were processed for TH immunohistochemical localization to determine the extent of dopaminergic innervation of the striatum during the first postnatal week. The above-described immu- 

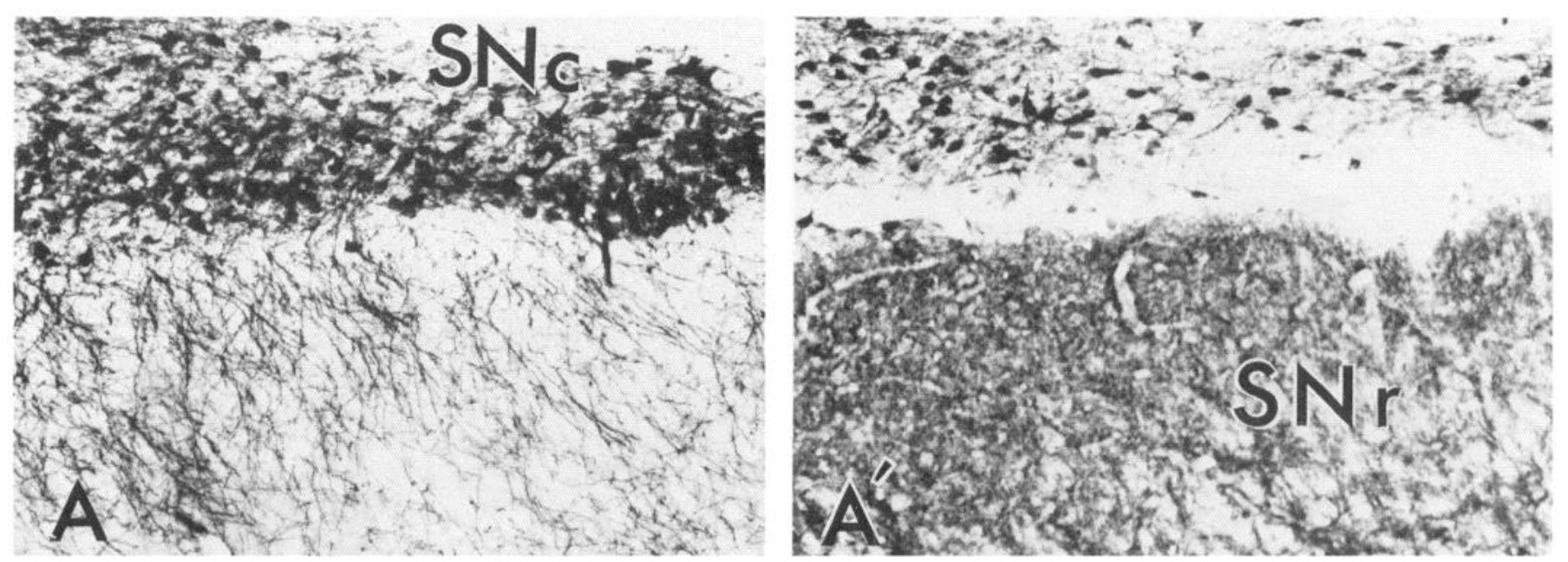

Figure 2. Higher magnification of Figure $1, A$ and $A^{\prime} . A$, TH-immunoreactive neurons in the SNc and the ventrally directed dendrites of ventral tier SNc neurons extending into the SNr. $A^{\prime}$, The same area from the adjacent section, labeled for CaBP immunoreactivity. Note that only dorsal tier SNc neurons express $\mathrm{CaBP}$ immunoreactivity, whereas ventral tier SNc neurons are unlabeled. Labeled dorsal tier neurons have dendrites extending mediolaterally within the plane of the SNc. CaBP immunoreactivity in terminals of matrix striatonigral efferents are distributed in the $\mathrm{SNr}$ and clearly avoid the location of the cell bodies of $\mathrm{SNc}$ neurons.

noperoxidase protocol was followed. These preliminary studies showed that, from birth through $\mathrm{P} 2$, TH immunoreactivity in the striatum is distributed primarily, but not exclusively, in the patch compartment. After this time, TH immunolabeling in the matrix increases dramatically, such that the borders between patch and matrix compartments with this label are not as easily recognized. Newborn rat pups, either $\mathrm{P} 0$ or P1, were taken from their mothers and hypothermically immobilized. A $2 \mu$ l solution of 6-OHDA $(2 \mathrm{mg} / \mathrm{ml})$ in saline plus $0.02 \%$ ascorbic acid was injected into the right striatum. The rat pups were revived and returned to their mothers. Injected rats were then perfused on P30 by the method described above and their brains processed for immunohistochemical localization of $\mathrm{TH}$ and $\mathrm{CaBP}$. Some sections at the level of the striatum were cut and immediately mounted onto chromalum-coated glass slides, air-dried, and stored frozen. These slidemounted sections were then processed for the autoradiographic localization of $\mu$-opiate receptors, using ${ }^{3} \mathrm{H}$-naloxone as a ligand (Herkenham and Pert, 1982) in perfusion-fixed tissue (Gerfen et al., 1985). In brief, slide-mounted sections were thawed and incubated in a solution containing $2.5 \mathrm{~nm}{ }^{3} \mathrm{H}$-naloxone (sp act, $44.4 \mathrm{Ci} / \mathrm{mmol}$; New England $\mathrm{Nu}$ clear) in $50 \mathrm{~mm}$ Tris buffer (pH 7.4) and $100 \mathrm{~mm} \mathrm{NaCl}$ at $4^{\circ} \mathrm{C}$ for 90 $\mathrm{min}$ and then rinsed 3 times for $1 \mathrm{~min}$ in Tris-buffered saline, air-dried, fixed in formaldehyde vapors $\left(80^{\circ} \mathrm{C}\right)$ for $90 \mathrm{~min}$, dehydrated, defatted in chloroform $/ \mathrm{methanol}$, and apposed to tritium-sensitive film $(3 \mathrm{H}$ Ultrofilm; LKB) for 3 weeks. The film was then developed in Kodak $\mathrm{D}-19\left(23^{\circ} \mathrm{C}\right.$ for $\left.5 \mathrm{~min}\right)$, rinsed, and air-dried.

\section{Results}

\section{Dopaminergic and CaBP-containing mesostriatal neurons}

Figure 1 shows adjacent coronal sections at rostral, mid, and caudal levels through the midbrain, labeled for $\mathrm{TH}$ and $\mathrm{CaBP}$ immunoreactivity. The A10 dopaminergic cell group, located medially in the ventral tegmental area through the rostrocaudal extent of the midbrain, is labeled with TH immunoreactivity (Fig. 1, A-C). A high percentage of neurons in this area are also labeled with $\mathrm{CaBP}$ immunoreactivity (Fig. 1, $A^{\prime}-C^{\prime}$ ). The A9 dopaminergic cell group, located in the substantia nigra pars compacta at all 3 levels (Fig. $1, A-C$ ) and in the ventral pars reticulata at mid and caudal midbrain levels (Fig. 1, B, C), is also labeled by $\mathrm{TH}$ immunoreactivity. Some of the pars compacta neurons have dendrites that extend ventrally into the pars reticulata; these are situated in a ventral tier of the pars compacta. CaBP immunoreactivity is located in only a subset of pars compacta neurons, those located in the dorsal tier. These
CaBP neurons are most prevalent at rostral (Fig. $1 A^{\prime}$ ) and mid (Fig. $\left.1 B^{\prime}\right)$ nigral levels and are distinctive not only in their dorsal location, but also in that their dendrites spread mediolaterally in the plane of the pars compacta and do not extend into the pars reticulata. Notably, few CaBP-immunoreactive neurons are located in the pars reticulata. CaBP is also localized in stri-

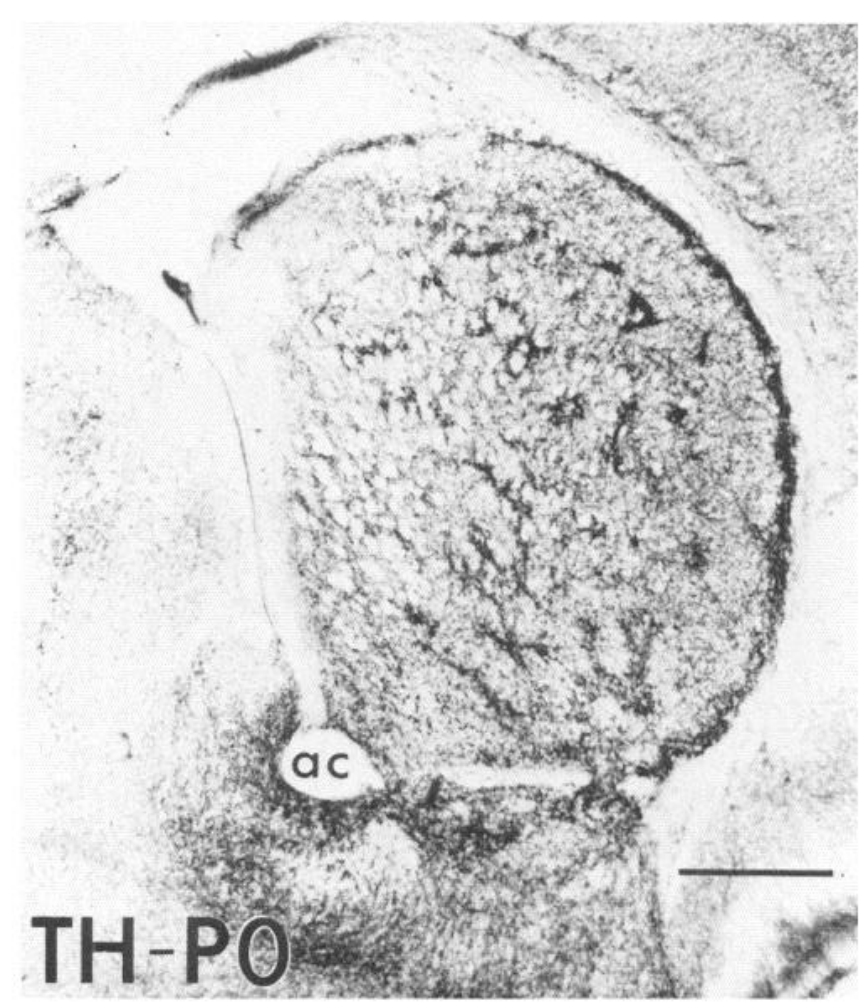

Figure 3. A coronal section through the striatum of a newborn rat $(\mathrm{P} 0)$ showing TH immunoreactivity. At this age, dopaminergic innervation is concentrated in the patches, with a markedly reduced input to the matrix compartment. Rats of this age received unilateral striatal injections of 6-OHDA. ac, Anterior commissure. Scale bar, $1 \mathrm{~mm}$. 

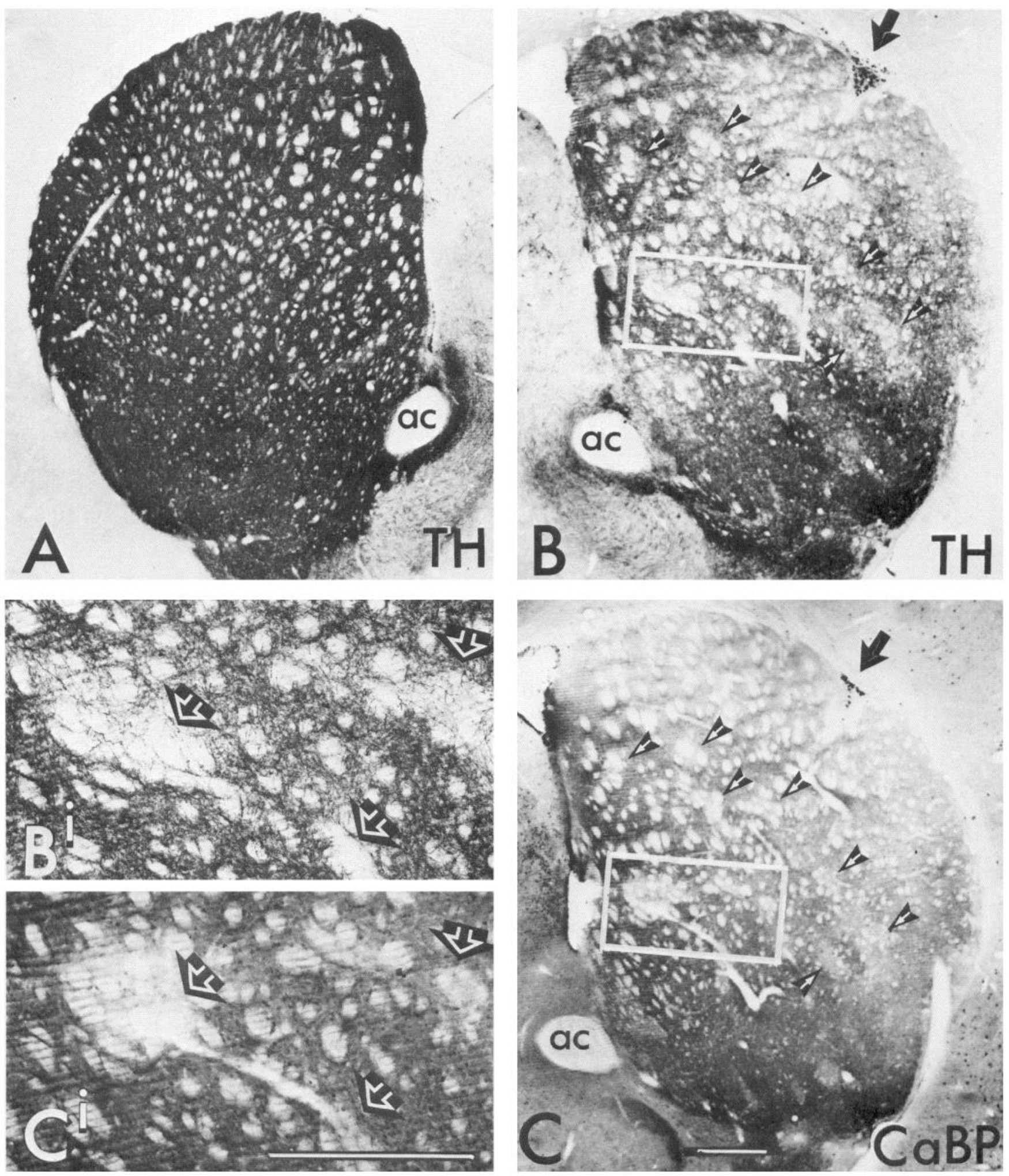

Figure 4. Striatal TH immunoreactivity is shown in a coronal section of a P30 rat contralateral $(A)$ and ipsilateral $(B)$ to an injection of 6-OHDA made at P0. The dense and homogenous pattern of TH immunoreactivity contralateral to the injection is typical of the adult pattern of striatal dopaminergic innervation. Corticofugal fiber fascicles are unlabeled. On the side of the lesion there are distinct patches of low density of TH-immunoreactive fibers $(B)$. These dopamine-denervated patches are aligned with patches containing few CaBP-immunoreactive neurons, shown in the adjacent section $(C)$. Arrows point to aligned patches of low TH fiber labeling $(B)$ and the corresponding patches of low CaBP immunoreactivity (C). An area containing 3 such aligned patches is shown at higher magnification in $B^{i}$ and $C^{\prime}$. Arrows point to the patches in both sections. The scar left by the needle tract is seen dorsolaterally in both $B$ and $C$ (large arrow). ac, Anterior commissure. Scale bar, $500 \mu \mathrm{m}$. 


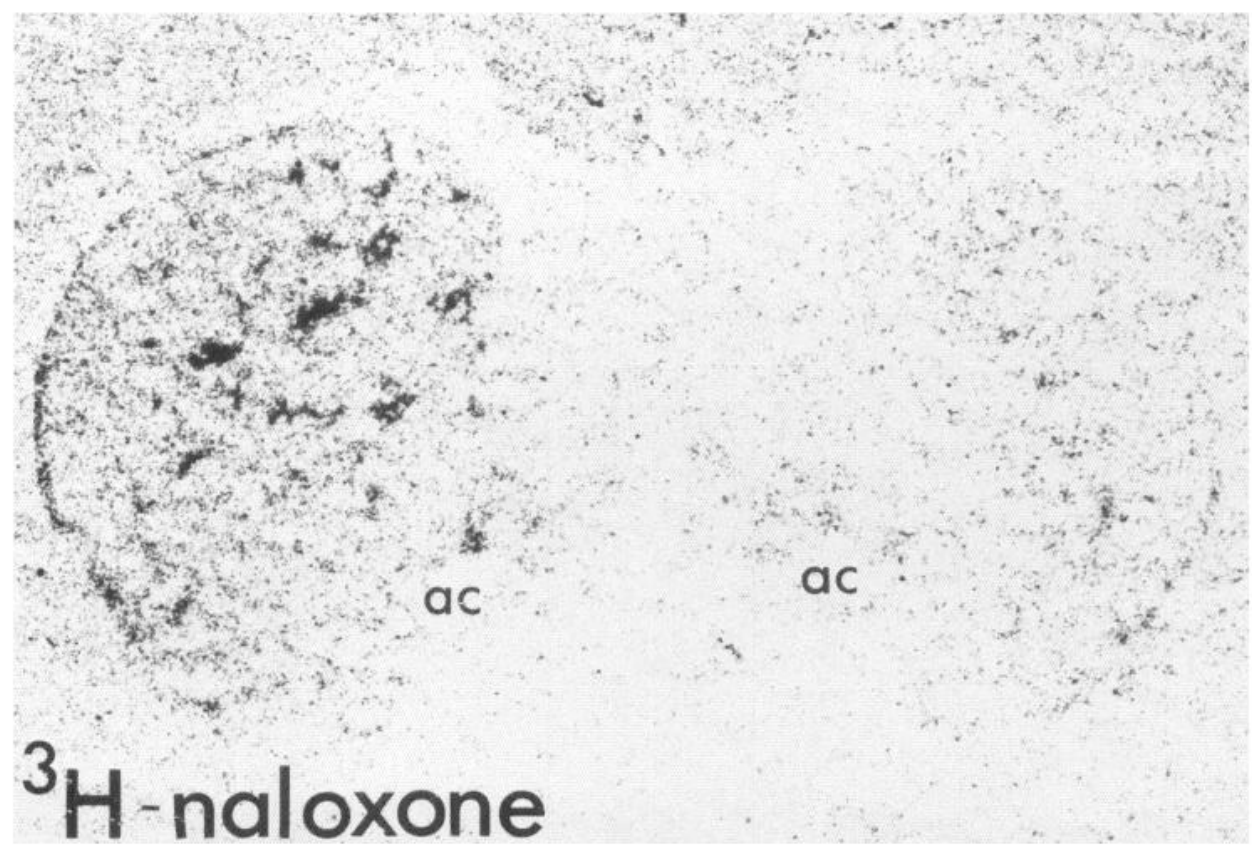

Figure 5. Photograph of an LKB autoradiographic film image of the distribution of ${ }^{3} \mathrm{H}$-naloxone binding to $\mu$-opiate receptor sites in a coronal section of the striatum adjacent to that shown in Figure 3, $A, B$. The left side is contralateral to the 6-OHDA injection and shows the typical pattern of distribution of dense ligand binding in the striatal patches. On the right side, which received the neonatal injection of 6-OHDA, there is a clear absence of increased binding in patches. $a c$, Anterior commissure. atonigral axon terminals distributed in the pars reticulata, and such terminals clearly avoid the location of dopaminergic neurons in both the pars compacta and the pars reticulata. Figure 2 shows a higher magnification of the central portion of the substantia nigra pars compacta and underlying pars reticulata of Figure 1, $A, A^{\prime}$. The A8 dopaminergic cell group is located at caudal levels (Fig. $1 C$ ) of the midbrain in the retrorubral area and is situated just dorsal to the pars compacta, above the medial lemniscus. These $\mathrm{TH}$-immunoreactive neurons have radially oriented dendrites and are relatively widely dispersed. CaBP immunoreactivity also labels many neurons in the retrorubral area (Fig. $1 C^{\prime}$ ).

\section{Neonatal 6-OHDA striatal lesions}

TH immunoreactivity in the striatum is patchy on $\mathrm{P} 0$, the day of birth (Fig. 3). The appearance of adult striatal TH immunoreactivity after unilateral lesions were made in the striatum by 6-OHDA administered on $\mathrm{P} 0$ is shown in Figure 4 . On the unlesioned side, TH immunoreactivity is dense and homogenous (Fig. 4A), typical of the striatum in normal adults. On the lesioned side, TH-labeled fibers are distributed heterogeneously, with distinct patches that contain relatively few fibers (Fig. 4, $\left.B, B^{i}\right)$. These areas of low fiber density correspond to the patches marked in adjacent sections by the relative absence of $\mathrm{CaBP}$ immunoreactive neurons (Fig. 4, C, $C^{i}$ ). The depletion of TH immunoreactivity in the patches was by no means absolute. There were some labeled fibers in the patches and there was also a decrease in fiber labeling in the matrix compartment relative to the unlesioned side. Patches are not to be confused with the unlabeled bundles of corticofugal fibers.

The distribution of $\mu$-opiate receptors was also examined in adjacent sections (Fig. 5). On the unlesioned side, the distinctive concentration of ${ }^{3} \mathrm{H}$-naloxone binding sites in the striatal patches is evident, whereas on the lesioned side there is a dramatic depletion of the binding in the patches.

The distribution of dopaminergic midbrain neurons, which project to the normal (Fig. 4, left) and lesioned (Fig. 4, right) striatum in the above-documented case is shown in Figure 6 and summarized quantitatively in Table 1 . At rostral midbrain levels (Fig. 6A), surviving dopaminergic neurons in the ventral tegmental area on the lesioned right side are only partially reduced in number compared to the unlesioned side. In the pars compacta there is a marked reduction in surviving neurons compared to the unlesioned side. Surviving neurons on the lesioned side are located in the dorsal tier of the pars compacta and have dendrites that spread mediolaterally in the plane of the nucleus. The neurons that are lost at this level from the A9 dopaminergic cell group are those located in the ventral tier of the pars compacta. The dendritic morphology of the lost neurons is inferred from the absence of ventrally directed dendrites into the pars reticulata, as compared on the unlesioned side. Only a few of these neurons remain on the lesioned side (see Table 1). At mid midbrain levels (Fig. 6B) there is a similar pattern of surviving dopaminergic neurons on the lesioned side. As at rostral levels, the ventral tegmental area shows only a slight reduction of neurons on the lesioned side. In the substantia nigra, most dorsal tier pars compacta neurons survive on the lesioned side, whereas there is a selective loss of most ventral tier pars compacta neurons that have dendrites extending into the pars reticulata. In addition, there is an almost complete loss of dopaminergic neurons in the pars reticulata. At caudal levels (Fig. 6C), there is again a relatively undiminished number of surviving dopaminergic neurons in the ventral tegmental area on the lesioned versus the unlesioned side. Similarly, there appears to be a relative sparing of retrorubral dopaminergic neurons on the lesioned side. At this level the most marked decrease in numbers of dopaminergic neurons is in the pars compacta and pars reticulata.

Whereas there is a loss of a set of dopaminergic neurons on the lesioned side of neonatally injected 6-OHDA rats, the relative number of CaBP-immunoreactive neurons in the midbrain is similar on the lesioned and unlesioned sides. Figure 7 shows an example of the distribution of CaBP-immunoreactive neu- 

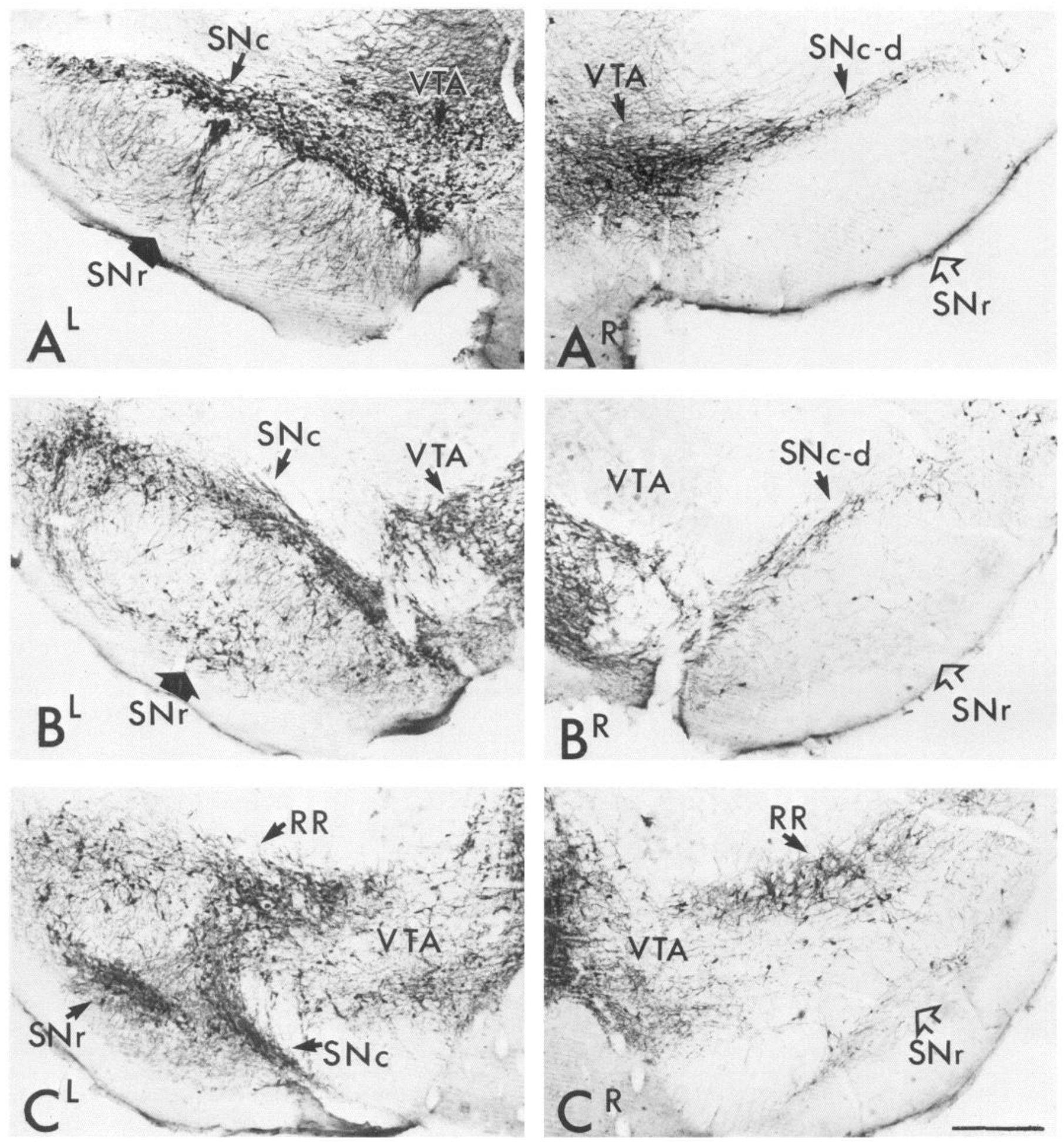

Figure 6. Photographs of coronal sections through rostral $(A)$, mid $(B)$, and caudal $(C)$ levels of the ventral midbrain showing the distribution of TH-immunoreactive cells contralateral $\left(A^{L}-C^{L}\right)$ and ipsilateral $\left(A^{R}-C^{R}\right)$ to the neonatal striatal injection of 6-OHDA from the same case documented in Figure 4. At rostral levels $(A)$, there is only a slight decrease in the number of surviving A10 dopaminergic neurons in the ventral tegmental area $(V T A)$, whereas those A9 cells surviving are distributed in the dorsal tier of the substantia nigra pars compacta $(S N c-d)$. These surviving A9 cells have dendrites extended within the plane of the SNc, whereas, by comparison with the contralateral side, most ventral tier A9 SNc neurons, with dendrites extended ventrally into the substantia nigra pars reticulata $(S N r)$, are selectively lost. At mid midbrain levels $(B)$, there is a similar pattern of survival of A10 and A9 dopaminergic neurons on the lesioned side $\left(B^{R}\right)$. At this level it is also evident that not only are most ventral $\mathrm{SNc}$ neurons lesioned, but also those dopaminergic neurons in the ventral SNr. At caudal midbrain levels $(C)$, both A10 and A8 dopaminergic cells survive on the lesioned side. At this level there is a dramatic loss of A9 neurons in both the SNc and SNr. Scale bar, $500 \mu \mathrm{m}$. 

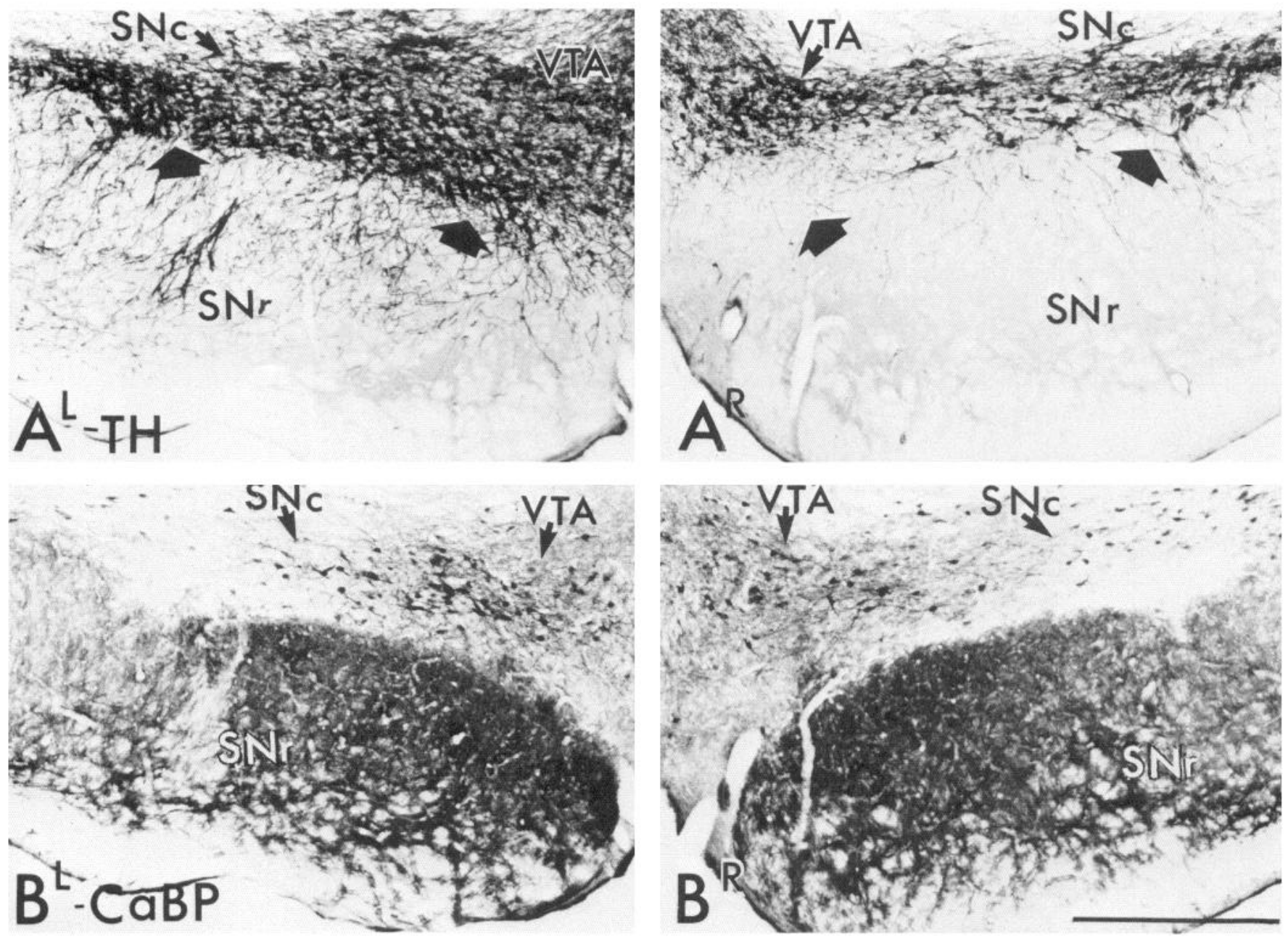

Figure 7. Adjacent coronal sections from a P30 rat through the rostral midbrain contralateral $\left(A^{L}, B^{L}\right)$ and ipsilateral $\left(A^{R}, B^{R}\right)$ to a neonatal striatal 6-OHDA injection showing the distribution of dopaminergic neurons labeled with TH immunoreactivity $(A)$ and CaBP-immunoreactive neurons $(B)$. Again, as shown in Figure 6, there is little reduction in the number of A10 dopaminergic neurons and the select loss of ventral tier, but not dorsal tier, A9 pars compacta neurons. However, there is little difference between the numbers of CaBP-immunoreactive neurons on the lesioned and the unlesioned side. Scale bar, $250 \mu \mathrm{m}$.

Table 1. Average number of cells labeled for TH or CaBP immunoreactivity per area in a 30- $\mu$ m-thick section from 5 P30 rats that had received a 6-OHDA injection into the right striatum on $\mathrm{P0}$

\begin{tabular}{|c|c|c|c|c|}
\hline & \multicolumn{2}{|l|}{ Left } & \multicolumn{2}{|l|}{ Right (lesioned) } \\
\hline & $\mathrm{TH}$ & $\mathrm{CaBP}$ & TH (\% of left) & $\mathrm{CaBP}(\%$ of left) \\
\hline VTA & $217 \pm 18$ & $156 \pm 17$ & $179 \pm 25(82)$ & $132 \pm 13(84)$ \\
\hline SNc-d & $79 \pm 8$ & $86 \pm 8$ & $54 \pm 8$ & $62 \pm 9$ \\
\hline SNc-v & $87 \pm 12$ & $9 \pm 6$ & $14 \pm 8 \quad(16)$ & $4 \pm 3$ \\
\hline $\mathrm{SNr}$ & $32 \pm 4$ & $2 \pm 2$ & $3 \pm 3$ & $1 \pm 1$ \\
\hline $\mathrm{RR}$ & $109 \pm 12$ & $89 \pm 10$ & $96 \pm 13(88)$ & $78 \pm 11(87)$ \\
\hline
\end{tabular}

These rats have a select depletion of TH immunoreactivity in the striatal patches on the lesioned side, similar to that shown in Figure 4. The pattern of TH immunoreactivity in the unlesioned left striatum and midbrain appears identical to that of normal rats. Cell counts for each area are taken, in each case, from 2 sections, from levels corresponding to those shown in Figure 6: ventral tegmental area (VTA), mid midbrain level; dorsal tier of the substantia nigra pars compacta (SNc-d), rostral level; ventral tier of the pars compacta (SNc-v), rostral level; substantia nigra pars reticulata $(\mathrm{SNr})$, mid midbrain; and retrorubral area (RR), caudal midbrain. The demarcation between the dorsal and ventral tier of the $\mathrm{SNc}$ is determined by the distribution of CaBP-containing neurons. rons in another case, which shows a similar reduction in striatal patch TH immunoreactivity. In this case there is a selective loss of ventral tier pars compacta dopaminergic neurons, which have ventrally directed dendrites, while dorsal tier pars compacta neurons are spared (Fig. $7 \mathrm{~A}$ ). In an adjacent section, the relative number and distribution of $\mathrm{CaBP}$-immunoreactive neurons in the ventral tegmental area and substantia nigra are similar on both sides (Fig. 7B). Throughout the rostrocaudal extent of the midbrain, including the retrorubral area, there is only a slight apparent difference between the distribution of CaBP-immunoreactive neurons on the lesioned and unlesioned sides. Table 1 provides cell counts of $\mathrm{TH}$ and $\mathrm{CaBP}$ neurons in various midbrain areas from 5 cases which had select depletions of $\mathrm{TH}$ in the patches.

\section{Discussion}

\section{CaBP labeling in matrix-directed mesostriatal neurons}

The $28 \mathrm{kDa}$ calcium-binding protein $(\mathrm{CaBP}$, or calbindin$\mathrm{D}_{28 \mathrm{kDa}}$ ), purified from human cerebellum and shown to be similar to a protein originally isolated from the chicken intestine 
that is induced by vitamin D (Baimbridge et al., 1982), has been shown to bc compartmentally distributed in the basal ganglia (Gerfen et al., 1985). In the striatum, CaBP is preferentially localized in neurons distributed in the matrix compartment, which project to the location of GABA neurons in the substantia nigra pars reticulata. This system is distinct from that arising from the striatal patch compartment, which projects to the location of dopaminergic neurons in the substantia nigra pars compacta and pars reticulata (Gerfen, 1984, 1985). Thus, the striatonigral patch and matrix systems are biochemically differentiated by CaBP. Additionally, we showed that CaBP is colocalized within a subset of dopaminergic (and non-dopaminergic) midbrain neurons in the ventral tegmental area, substantia nigra pars compacta, and retrorubral area (Gerfen et al., 1985). The present study examines the distribution of these midbrain CaBP neurons in more detail. Their distribution coincides with that of midbrain neurons projecting to the striatal matrix compartment (Gerfen et al., 1987). Figure 1 shows the distribution of CaBP neurons, which, when compared with the distribution of neurons that project to the striatal matrix (Figs. 11,12 in Gerfen et al., 1987), demonstrates the coincidence in patterns of distribution.

\section{Developmental dissociation of patch and matrix nigrostriatal dopaminergic systems}

In the present study, advantage was taken of the asynchronous development of striatal dopaminergic innervation by injecting 6-OHDA into the striatum at a time when this input was welldeveloped in the patches but not in the matrix (P0). Such lesions result in the relatively selective and long-lasting depletion of dopaminergic innervation of the striatal patch compartment (Fig. 3). Two pieces of evidence support the interpretation that the dopaminergic innervation to the patches is selectively lesioned by this manipulation. First, there is a direct correspondence between the patches of low density of TH-immunoreactive fibers on the lesioned side and those of low density of CaBP-immunoreactive neurons, which have previously been shown to be a consistent marker for the striatal matrix (Gerfen et al., 1985). Second, there is a selective loss of the high-density binding of ${ }^{3} \mathrm{H}$-naloxone in striatal patches on the lesioned side. Thus, whereas cells in the patches are intact, there is a selective absence of 2 biochemical markers in the patches, TH-immunoreactive fibers and $\mu$-opiate receptor binding sites. This latter finding warrants further study, as it would appear to confirm earlier reports that such receptor sites may be localized presynaptically on dopaminergic afferents (Murrin et al., 1980; Bowen et al., 1986; but see Hattori and Fibiger, 1982).

The selective depletion of dopaminergic inputs to the patches is by no means absolute. There is a decrease in fiber labeling in both compartments relative to the unlesioned side. However, there is clearly a greater loss of fibers in the patches. This is to be expected since, at the time of the lesions on $\mathrm{P} 0$, while there is a far greater presence of dopaminergic afferents in the patches, there are also some afferents in the matrix. Presumably striatal 6-OHDA lesions on P0 denervate both systems, but have a greater effect on the patch innervation system. This result fortuitously provides a means for comparing the distribution of surviving midbrain dopaminergic neurons that provide inputs to the striatal matrix with that predicted from the preceding neuroanatomical study (Gerfen et al., 1987) and from the present results concerning the distribution of CaBP-containing dopaminergic neurons, which have been suggested to project to the striatal matrix. The results of the neonatal 6-OHDA striatal lesions appear to confirm both predictions in the following manner. Dopaminergic neurons selectively lost by this procedure are those in the ventral tier of the pars compacta and in the substantia nigra pars reticulata, which were shown in the preceding study to project to the striatal patches (Gerfen et al., 1987). Dopaminergic neurons surviving this procedure are distributed in the ventral tegmental area, the dorsal tier of the substantia nigra pars compacta, and in the retrorubral area. These surviving neurons also appear to express CaBP, whereas the ones that do not are selectively lost with the concomitant depletion of dopaminergic inputs to the striatal patches.

These results confirm an additional point concerning the morphology of the neurons that project to the patch and matrix compartments. Substantia nigra neurons that project to the patches, those lost by neonatal 6-OHDA striatal lesions, have dendrites that extend into the pars reticulata, whereas the neurons that survive this procedure, which are located dorsally and project to the matrix, have dendrites that are extended mediolaterally in the plane of the pars compacta. Fallon et al. (1978) described a similar morphologic dissociation of dorsal and ventral tier midbrain dopaminergic neurons. It might be argued that the present data, based on the distribution of dorsal tier neurons surviving neonatal 6-OHDA lesions, reflect the survival of neurons projecting to cortical areas, which are unaffected by the lesions. However, the preceding neuroanatomical study, using PHA-L anterograde tracing, clearly demonstrated the projections from dorsal tier neurons to the striatal matrix compartment. Furthermore, it has been shown that pars compacta neurons have collaterals that are distributed both to the striatum and to nonstriatal telencephalic areas, including the septum and cortex (Fallon and Loughlin, 1982; Loughlin and Fallon, 1984; Takada and Hattori, 1986).

\section{Functional implications}

The categorization of separate mesolimbic and nigrostriatal dopaminergic systems serves a useful role in attempts to determine the manner in which these systems regulate behavior. The nigrostriatal system, providing inputs to the caudate and putamen, is clearly related, at least in part, to motor functions, as evidenced by the characteristic movement disorders associated with its degeneration in Parkinson's disease (Hornykiewicz, 1966). The mesocorticolimbic dopaminergic system, innervating the ventral striatum, cortex, and limbic areas, is thought to be related to mood (Heimer and Wilson, 1975; Nauta et al., 1978; Fibiger, 1984; Fibiger and Phillips, 1986). Dysfunction of this system has been hypothesized to underlie the etiology of schizophrenia and depression (Stevens, 1973). Numerous behavioral studies have suggested that the mesolimbic dopaminergic pathways are substrates for motivation and reward (see Fibiger, 1984, and Fibiger and Phillips, 1986, for review). Pharmacological manipulation points to the interrelation of dopaminergic affects on movement and mood (see Creese, 1983, and Penney and Young, 1983). For example, L-DOPA therapy for the alleviation of Parkinsonian movement disorders may have profound mood-altering effects and lead in some instances to psychotic episodes. Conversely, antipsychotic dopamine receptor-blocking agents, in many cases of long-term use, result in significant movement disorders, such as tardive dyskinesia. Recently, certain dopamine antagonists, the so-called "atypical neuroleptics," have been shown to be effective as antipsychotic drugs without producing dyskinesias (see Creese, 1983). Phys- 
iological studies suggest that such drugs have different effects on the A10 mcsolimbic and A9 nigrostriatal projection systems (Chiodo and Bunney, 1983; White and Wang, 1983). These data are consistent with concepts of the functional dissociation of striatal subregions (Heimer and Wilson, 1975; Nauta et al., 1978; Kelley et al., 1982).

Given the present findings that midbrain dopaminergic neurons are anatomically and biochemically parcelled into dorsal and ventral tier sets, rather than by their inclusion in the A10 and $A 9$ cell groups, a reevaluation of the pharmacologic effects of neuroleptics on midbrain dopamine neurons is required. There is already considerable evidence suggesting that both A10 and A9 cell groups are pharmacologically and physiologically heterogeneous in terms of their discharge patterns and sensitivity to autoreceptor regulation. In the ventral tegmental area, slowfiring A10 dopamine neurons are more sensitive to the effects of dopamine agonist action on autoreceptors than are fast-firing cells (Chiodo et al., 1984; Shepard and German, 1984; Whitc and Wang, 1984; Clark et al., 1985). Furthermore, A10 neurons projecting to the prefrontal and cingulate cortex lack autoreceptors, whereas A10 neurons projecting to the pyriform cortex possess such autoreceptors (Chiodo et al., 1984). Similarly, Shepard and German (1987a, b) have reported the existence of 2 sets of nigrostriatal neurons, distinguished by a correlation between their discharge patterns and their sensitivity to autureceptor regulation. It would be of interest to determine whether the 2 neuroanatomically and biochemically distinct patch and matrix mesostriatal systems are correlated with physiologically distinct types of neurons. Such a correlation would suggest that the differential effects of typical and atypical neuroleptics on movement and mood, and the interrelationship between these functions, may be related to the compartmental organization of mesostriatal systems.

The manner in which pharmacological treatments may differentially affect the dual mesostriatal dopaminergic systems is now being examined. After pharmacological treatments that deplete striatal dopamine stores in adult rats, there is a more rapid replenishment of dopamine in patches than in the matrix (Olson et al., 1972; Fuxe et al., 1979; Fukui et al., 1986). Using in situ hybridization techniques, Young et al. (1986) have shown that mesostriatal dopaminergic systems affcct the expression of striatal peptide levels, demonstrating that 6-OHDA lesions of the entire system result in an up-regulation of enkephalin mRNA expression and a down-regulation of substance $P$ mRNA expression. Chronic apomorphine treatment of rats causes a selective increase in dynorphin immunoreactivity in the striatonigral patch system (Li et al., 1986), despite the expression of dynorphin in both the patch and matrix striatonigral systems (Young et al., 1986; C. R. Gerfen and S. J. Young, unpublished observations). Such studies provide the means for examining the differential effects of pharmacologic manipulation on the patch-and matrixdirected mesostriatal dopaminergic systems.

The present results provide further evidence for the existence of separate mesostriatal systems that differentially innervate the striatal patch and matrix compartments. In the preceding paper (Gerfen et al., 1987), the relationship of this system to the compartmentally organized striatonigral system (Gerfen, 1984, 1985; Gerfen et al., 1985) was discussed in terms of the integration of movement and mood by the basal ganglia. How this organization is manifested in terms of the neurochemical regulation of behavior is currently under investigation. Much attention has been focused on the role of the transmitters and peptides used in these systems. However, the finding of a dissociation of the compartmentally organized striatonigral (Gerfen et al., 1985) and mesostriatal systems on the basis of the expression of $\mathrm{CaBP}$ suggests a need to examine other aspects of the biochemical phenotype of these neurons to determine the mechanism by which the physiologic patterns in complexly organized neural circuits are regulated. For example, calcium-dependent mechanisms appear to underlie the physiologic basis of irregular and bursting dopaminergic neurons (Grace and Bunney, 1984a, b) and the regulation of dendritic release of dopamine from ventral tier pars compacta neurons (Llinas et al., 1984). Whether the expression of $\mathrm{CaBP}$ by dorsal and not ventral tier mesostriatal dopaminergic neurons is related to these physiologic mechanisms is of interest. Similarly, in the striatum, patch and matrix medium spiny neurons share many of the same peptides and transmitters, including GABA, dynorphin, substance $P$, and enkephalin (Aronin et al., 1984; Penny et al., 1986; Young et al., 1986), but are dissociatcd by the expression in matrix neurons of CaBP. Elucidating the manner in which such a protein is related to the physiological and pharmacologic regulation of neuronal activity and of peptide expression will provide additional insights to the functional organization of the basal ganglia.

\section{References}

Arluison, M., M. Dietl, and J. Thibault (1984) Ultrastructural morphology of dopaminergic nerve terminals and synapses in the striatum of the rat using tyrosine hydroxylase immunoreactivity: A topographical study. Brain Res. Bull. 13: 269-285.

Aronin, N., M. Difiglia, G. A. Graveland, W. J. Schwartz, and J.-Y. Wu (1984) Localization of immunoreactive enkephalins in GABA synthesizing neurons of the rat neostriatum. Brain Res. 300: 376380.

Baimbridge, K. G., J. J. Miller, and C. O. Parkes (1982) Calciumbinding protein distribution in rat brain. Brain Res. 239: 519-525.

Bowen, W. D., C. B. Pert, and A. Pert (1986) Nigral 6-hydroxydopamine lesions equally decrease $\mu$ and $\delta$ opiate binding to striatal patches: Further evidence for a conformationally malleable type I opiate receptor. Life Sci. 31: 1679-1682.

Butcher, L. L., and G. K. Hodge (1976) Postnatal development of acetylcholinesterase in the caudate-putamen and substantia nigra of rats. Brain Res. 106: 223-240.

Chiodo, L. A., and B. S. Bunney (1983) Typical and atypical neuroleptics: Differential effects of chronic administration on the activity of A9 and A10 midbrain dopaminergic neurons. J. Neurosci. 3: $1607-$ 1619.

Chiodo, L. A., M. J. Bannon, A. A. Gracc, R. H. Roth, and B. S. Bunney (1984) Evidence for the absence of impulse-regulating somatodendritic and synthesis-modulating nerve terminal receptors on subpopulations of mesocortical dopamine neurons. Neuroscience 12:1-16.

Clark, D., G. Enberg, E. Peleblan, T. H. Svennon, and A. Carlsson (1985) An electrophysiological analysis of the actions of the 3-PPP enantiomers on the nigrostriatal dopamine system. Nauyn Schmiedebergs Arch. Pharmacol. 329: 344-354.

Creese, I. (1983) Classical and atypical antipsychotic drugs: New insights. Trends Neurosci. 6: 479-481.

Fallon, J. H., and S. E. Loughlin (1982) Monoamine innervation of the forebrain: Collateralization. Brain Res. Bull. 9: 295-307.

Fallon, J. H., J. N. Riley, and R. Y. Moore (1978) Substantia nigra dopamine neurons: Separate populations project to neostriatum and allocortex. Neurosci. Lett. 7: 157-162.

Fibiger, H. C. (1984) The neurological substrates of depression in Parkinson's disease: A hypothesis. Can. J. Neurol. Sci. 11: 105-107.

Fibiger, H. C., and A. G. Phillips (1986) Reward, motivation, cognition: Psychobiology of mesotelencephalic dopamine systems. In Handbook of Physiology, The Nervous System, vol. IV: Intrinsic Regulatory Systems of the Brain, F. E. Bloom, ed., pp. 647-675, American Physiological Society, Baltimore, MD.

Fukui, K., H. Kariyama, A. Kashiba, N. Kato, and H. Kimura (1986) Further confirmation of heterogeneity of the rat striatum: Different 
mosaic patterns of dopamine fibers after administration of meth amphetamine or reserpine. Brain Res. 382:81-86.

Fuxe, K., K. Andersson, R. Schwarcz, L. F. Agnati, M. Perez de la Mora, T. Hökfelt, M. Goldstein, L. Ferland, L. Possani, and R. Tapia (1979) Studics on different types of dopamine nerve terminals in the forebrain and their possible interactions with hormones and with neurons containing GABA, glutamate, and opioid peptides. In $A d$ vances in Neurology, vol. 24 , L. J. Poirier, T. L. Sourkes, and P. J. Bedard, eds., pp. 199-215, Raven, New York.

Gerfen, C. R. (1984) The neostriatal mosaic: Compartmentalization of corticostriatal input and striatonigral output systems. Nature 311 : $461-464$

Gerfen, C. R. (1985) The neostriatal mosaic: I. Compartmental organization of projections from the striatum to the substantia nigra in the rat. J. Comp. Neurol. 236: 454-476.

Gerfen, C. R., K. G. Baimbridge, and J. J. Miller (1985) The neostriatal mosaic: Compartmental distribution of calcium binding protein and parvalbumin in the basal ganglia of the rat and monkey. Proc. Natl. Acad. Sci. USA 82: 8780-8784.

Gerfen, C. R., M. Herkenham, and J. Thibault (1987) The neostriatal mosaic: II. Patch-and matrix-directed mesostriatal dopaminergic and non-dopaminergic systems. J. Neurusci. 7: 3915-3934.

Grace, A. A., and B. S. Bunney (1984a) The control of firing pattern in nigral dopamine neurons: Single spike firing. J. Neurosci. 4:28662876.

Grace, A. A., and B. S. Bunney (1984b) The control of firing pattern in nigral dopamine neurons: Burst firing. J. Neurosci. 4: 2877-2890.

Graybiel, A. M. (1984) Correspondence between the dopamine islands and striosomes of the mammalian striatum. Neuroscience 13: 11571187.

Graybiel, A. M., V. M. Pickel, T. H. Joh, D. J. Reis, and C. W. Ragsdale, Jr. (1981) Direct demonstration of a correspondence between the dopamine islands and acetylcholinesterase patches in the developing striatum. Proc. Natl. Acad. Sci. USA 78: 5871-5875.

Hattori, T., and H. C. Fibiger (1982) On the use of lesions of afferents to localize neurotransmilter receptor sites in the striatum. Brain Res. 238: $245-250$.

Heimer, L., and R. D. Wilson (1975) The subcortical projections to the allocortex: Similarities in the neural associations of the hippocampus, the piriform cortex and the neocortex. In Golgi Centennial Symposium, M. Santini, ed., pp. 177-193, Raven, New York.

Herkenham, M., and C. B. Pert (1982) Light microscopic localization of brain opiate receptors: A general autoradiographic method which preserves tissue quality. J. Neurosci. 2: 1129-1149.

Hornykiewicz, O. (1966) Dopamine (3-hydroxytryptamine) and brain function. Pharmacol. Rev. 18: 925-964.

Kelley, A. E., V. B. Domesick, and W. J. H. Nauta (1982) The amygdalostriatal projection in the rat - an anatomical study by anterograde and retrograde tracing methods. Neuroscience 7: 615-630.

Li, S., S. P. Sivam, J. F. McGinty, and J. S. Ilong (1986) Regulation of the metabolism of opioid peptides in the striatonigral pathway by the dopaminergic system. Soc. Neurosci. Abstr. 12: 1044.

Llinas, R., S. A. Greenfield, and H. Jahnsen (1984) Electrophysiology of pars compacta cells in the in vitro substantia nigra-a possible mechanism for dendritic release. Brain Res. 294: 127-132.
Loughlin, S. E., and J. H. Fallon (1984) Substantia nigra and ventral tegmental area projections to cortex: Topography and collateralization. Neuroscience 11: 425-435.

Moon-Edley, S., and M. Herkenham (1984) Comparative development of striatal opiate receptors and dopamine revealed by autoradiography and histofluorescence. Brain Res. 305: 27-42.

Murrin, L. C., J. T. Coyle, and M. J. Kuhar (1980) Striatal opiate receptors: Pre- and posisynaptic localization. Life Sci. 27: 1175-1 183.

Nauta, W. J. H., G. P. Smith, R. L. M. Faull, and V. B. Domesick (1978) Efferent connections and nigral afferents of the nucleus accumbens septi in the rat. Neuroscience 3: 385-401.

Olson, L., A. Seiger, and K. Fuxe (1972) Heterogeneity of striatal and limbic dopamine innervation: Highly fluorescent islands in developing and adult rats. Brain Res. 44: 283-288.

Penney, J. B., Jr., and A. B. Young (1983) Speculations on the functional anatomy of hasal ganglia disorders. Annu. Rev. Neurosci. 6: 73-94.

Penny, G. R., S. Afsharpour, and S. T. Kitai (1986) The glutamic acid decarboxylase-, leucine-, enkephalin-, and substance $\mathrm{P}$-immunoreactive neurons in the neostriatum of the rat and cat: Evidence for partial population overlap. Neuroscience 17: 1011-1045.

Shepard, P. D., and D. C. German (1984) A subpopulation of mesocortical dopaminergic neurons possess autoreceptors. Eur. J. Pharmacol. 98: 455-456.

Shepard, P. D., and D. C. German (1987a) Regional differences in substantia nigra neurons: Electrophysiological properties. Neuroscience (in press).

Shepard, P. D., and D. C. German (1987b) Regional differences in substantia nigra dopamine neurons: Pharmacological properties. Neuroscience (in press).

Stevens, J. R. (1973) An anatomy of schizophrenia? Arch. Gen. Psychiatry 29: 177-189.

Takada, M., and T. Hattori (1986) Collateral projections from the substantia nigra to the cingulate cortex and striatum in the rat. Brain Res. 380: 331-335.

Tennyson, V. M., R. E. Barrett, G. Cohen, L. Cote, R. IIeikkila, and C. Mytilineou (1972) The developing neostriatum of the rabbit: Correlation of fluorescence histochemistry, electron microscopy, endogenous dopamine levels, and $(3 \mathrm{H})$ dopamine uptake. Brain Res. 46 : 251-285.

van der Kooy, D. (1984) Developmental relationships between opiate receptors in the formation of the caudate-putamen patches. Dev. Brain Res. 14: 300-303.

White, F. J., and R. Y. Wang (1983) Differential effects of classical and atypical antipsychotic drugs on $\mathrm{A} 9$ and $\mathrm{A} 10$ dopamine neurons. Science 221: 1054-1057.

White, F. J., and R. Y. Wang (1984) A10 dopamine neurons: Role of autoreceptors in determining firing rate and sensitivity to dopamine agonists. Life Sci. 34: 1161-1170.

Young, W. S., III, T. I. Bonner, and M. R. Brann (1986) Mcsenccphalic dopamine neurons regulate the expression of neuropeptide mRNAs in the rat forebrain. Proc. Natl. Acad. Sci. USA 83: 9827-9831. 